PROCEEDINGS OF THE

AMERICAN MATHEMATICAL SOCIETY

Volume 135, Number 9, September 2007, Pages 2785-2793

S 0002-9939(07)08822-3

Article electronically published on March 30, 2007

\title{
UNIQUENESS FOR BOUNDARY BLOW-UP PROBLEMS WITH CONTINUOUS WEIGHTS
}

\author{
JORGE GARCÍA-MELIÁN
}

(Communicated by David S. Tartakoff)

\begin{abstract}
In this paper, we prove that for $p>1$ the problem $\Delta u=a(x) u^{p}$ in a bounded $C^{2}$ domain $\Omega$ of $\mathbb{R}^{N}$ has a unique positive solution with $u=\infty$ on $\partial \Omega$. The nonnegative weight $a(x)$ is continuous in $\bar{\Omega}$, but is only assumed to verify a "bounded oscillations" condition of local nature near $\partial \Omega$, in contrast with previous works, where a definite behavior of $a$ near $\partial \Omega$ was imposed.
\end{abstract}

\section{INTRODUCTION AND RESULTS}

This work is concerned with the uniqueness issue of positive solutions to the boundary blow-up elliptic problem

$$
\left\{\begin{array}{ccc}
\Delta u=a(x) u^{p} & \text { in } \quad \Omega, \\
u=+\infty & \text { on } & \partial \Omega
\end{array}\right.
$$

where $p>1, \Omega$ is a bounded $C^{2}$ domain of $\mathbb{R}^{N}$ and $a \in C(\bar{\Omega})$ is a nonnegative weight function. As usual in problems of this type, the boundary condition is to be understood as $u(x) \rightarrow+\infty$ as $d(x)=\operatorname{dist}(x, \partial \Omega) \rightarrow 0+$.

This particular problem has been widely considered in the recent literature. Its study seems to have been started in [21, where the case $a(x)=1, p=(N+2) /$ $(N-2)$ was analyzed, and later continued in [19, [1], 2], 25], 24] (see also [10] for the $p$-Laplacian analogue), where $a(x) \geq a_{0}>0$ in $\bar{\Omega}$ and a linear term $-\lambda u$ is added in the equation in some cases. In all of them, uniqueness was obtained by means of precise boundary estimates, which took the form $u \sim A d^{-\alpha}$ as $d \rightarrow 0$, where $A$ is given in terms of $p$ and $\alpha=2 /(p-1)$. We refer the interested reader to [13] for an extensive list of references with more general nonlinearities $f(u)$ in (1.1). We only mention the pioneering works [3] and [17.

Some time later, this problem was reconsidered to allow more general weights $a(x)$. In the first place, they were permitted to vanish on $\partial \Omega$, with a precise rate of the form $a \sim C d^{\gamma}$, for positive constants $C, \gamma$, for instance in [12] and [14] (the constants $C$ and $\gamma$ were replaced by positive continuous functions in 22, and a further generalization to the $p$-Laplacian setting was considered in [11]). Uniqueness was also obtained there by using estimates of all possible solutions near the boundary (the second term in the asymptotic behavior of the solution near $\partial \Omega$ was also obtained in [14, extending previous results of 9]).

Received by the editors May 8, 2006.

2000 Mathematics Subject Classification. Primary 35J25; Secondary 35J60.

Supported by MEC and FEDER under grant MTM2005-06480.

(c)2007 American Mathematical Society Reverts to public domain 28 years from publication 
The next step was allowing the weight $a(x)$ to be singular on $\partial \Omega$. In this regard, problem (1.1) was analyzed in [26], under the assumption $a(x) \leq C d(x)^{-\gamma}$, for some $\gamma \in(0,2)$, although only existence was shown there. This study was completed later to also achieve uniqueness and global estimates in [5], complementing previous results in the radial case obtained in 4]. The essential hypothesis on the weight there was

$$
C_{1} d(x)^{-\gamma} \leq a(x) \leq C_{2} d(x)^{-\gamma}, \quad x \in \Omega,
$$

for positive constants $C_{1}, C_{2}$ and $\gamma \in(0,2)$.

We remark at this point that, although the existence issue has been clarified to allow very general (nonnegative) weights $a(x)$ which can even vanish in points of $\Omega$ (see 20]; on the other hand it is known that no solutions exist if $a$ vanishes in a neighborhood of a point on the boundary; see 15]), the uniqueness is not completely understood. More precisely, assume (1.1) admits a positive solution. The important question is: which is the optimal condition on $a(x) \in C(\Omega)$ so that the solution is unique?

Some work on this direction has recently been done, although only for weights verifying $a \in C(\bar{\Omega})$. An improvement in the previous boundary behavior of $a(x)$ was obtained in [7] and [8]. It was shown in [8] that if

$$
a(x)=k^{2}(d(x))+o\left(k^{2}(d(x))\right), \quad \text { as } d \rightarrow 0,
$$

where $k \in C^{1}(0, \delta)$ for some $\delta>0$ and

$$
\lim _{t \rightarrow 0} \frac{1}{k(t)} \int_{0}^{t} k(s) d s=0, \quad \lim _{t \rightarrow 0}\left(\frac{1}{k(t)} \int_{0}^{t} k(s) d s\right)^{\prime}=\ell_{1} \in[0,1],
$$

then (1.1) admits a unique positive solution, and its boundary behavior was precisely determined. The exact behavior (1.3) was later relaxed in [6] to a condition similar to (1.2), namely

$$
C_{1} k^{2}(d(x)) \leq a(x) \leq C_{2} k^{2}(d(x)),
$$

and $k$ still verifying (1.4). Uniqueness also holds in this case.

A further step was given in [23: if $a>0$ in $\Omega$ is such that $a \in C(\bar{\Omega}) \cap C^{1}\left(\bar{\Omega}_{\delta}\right)$ for some $\delta>0$, where $\Omega_{\delta}=\{x \in \Omega: 0<d(x)<\delta\}$, and the functions $f_{x}(t)=$ $a(x-t \nu(x))$, where $\nu(x)$ is the outward unit normal and $x \in \partial \Omega$, verify $f_{x} \in C^{2}(0, \delta]$, $f_{x}(0)=0, f_{x}^{\prime}(t)>0$ and $\left(\log f_{x}\right)^{\prime \prime}<0$ for each $t \in(0, \delta)$, while

$$
\lim _{\substack{x \rightarrow x_{0} \\ t \downarrow 0}} \frac{f_{x}(t)}{f_{x_{0}}(t)}=1
$$

uniformly on $\partial \Omega$, then (1.1) admits a unique positive solution, whose boundary behavior can be explicitly given.

Our intention in the present work is to give a simple sufficient condition on $a(x)$ to ensure uniqueness of the positive solution. Our proof does not make use of boundary estimates, but of a refinement of an iterative procedure due to Safonov, which has been previously used in [18, [5, [11] and [13]. This is why we do not need a definite behavior of $a(x)$ near $\partial \Omega$, nor do we require any further regularity than continuity. First we are assuming that $a>0$ in $\Omega_{\delta}$ for some small positive $\delta$ (this condition is enough to obtain existence; see [15] and [20]), and we are imposing a "bounded oscillations" property on $a(x)$, which can be stated as follows: 
Condition (A): For every $x_{0} \in \partial \Omega$, there exists a positive constant $C$ such that for every two sequences $\xi_{n}, \eta_{n} \subset \bar{\Omega}$ with $\xi_{n}, \eta_{n} \rightarrow x_{0}$, $a\left(\eta_{n}\right)>0$ and $d\left(\xi_{n}\right) / d\left(\eta_{n}\right)$ bounded, it holds that

$$
\frac{a\left(\xi_{n}\right)}{a\left(\eta_{n}\right)} \leq C .
$$

We also remark that the regularity of the domain is not essential to obtain our theorem, and that an exterior sphere condition would suffice. However, we have preferred to stay in the $C^{2}$ setting to make the proofs more clear.

After introducing our main hypotheses, we come to the statement of the results. Observe that, thanks to standard regularity theory for elliptic equations, positive weak solutions $u$ to (1.1) belong to $W_{\text {loc }}^{2, q}(\Omega)$ for all $q>1$, and the Sobolev imbedding gives $u \in C^{1, \nu}(\Omega)$ for all $\nu \in(0,1)$ (cf. [16]). Therefore, they are indeed strong solutions.

Theorem 1. Assume $\Omega$ is a bounded $C^{2}$ domain of $\mathbb{R}^{N}$, and $a \in C(\bar{\Omega})$ is strictly positive in $\Omega_{\delta}$ for some $\delta>0$ and satisfies condition (A). Then problem (1.1) has a unique positive strong solution $u \in C^{1, \nu}(\Omega) \cap W_{\mathrm{loc}}^{2, q}(\Omega)$ for every $\nu \in(0,1), q>1$.

The essential ingredient to prove Theorem 1 is the obtention of local estimates of the solutions in terms of a negative power of the distance $d(x)$. We achieve them by a rescaling argument similar to the one used in [5], by comparing the solutions with solutions in balls and annuli.

These estimates also give bounds for the boundary behavior of the solution without further restrictions on $a(x)$. We believe that this behavior cannot be completely elucidated unless some stronger conditions are placed on $a$.

Theorem 2. Assume $\Omega$ is a bounded $C^{2}$ domain of $\mathbb{R}^{N}$, and $a \in C(\bar{\Omega})$ is strictly positive in $\Omega_{\delta}$ for some $\delta>0$ and satisfies condition $(A)$. Then the unique solution $u$ to (1.1) verifies

$$
C_{1} a(x)^{-\frac{1}{p-1}} d(x)^{-\alpha} \leq u(x) \leq C_{2} a(x)^{-\frac{1}{p-1}} d(x)^{-\alpha}
$$

for $x \in \Omega_{\delta / 2}$, where $\alpha=2 /(p-1)$, and $C_{1}, C_{2}$ are positive constants.

Remarks 1. (a) We point out that the weight $a(x)$ is not required to vanish identically on $\partial \Omega$, as in most of the previous results in the literature (for instance 6 ] or [23]). However, condition (A) is only meaningful at a point $x_{0} \in \partial \Omega$ when $a\left(x_{0}\right)=0$; otherwise it is trivially satisfied.

(b) The uniqueness result contained in Theorem 1 is complementary to those in 6] or [23]. However, it has the advantage to require mere continuity to the weight $a(x)$.

(c) We remark that condition (A) is not optimal. Indeed, there are functions like $a(x)=e^{-d(x)^{-\gamma}}$ for which uniqueness holds thanks to Theorem 1.1 in [6], but do not verify condition $(\mathrm{A})$.

(d) The proof of Theorem 1 can be extended to cover some more general nonlinearities $f(u)$, as in [13. It suffices that $f(u) \sim u^{p}$ as $u \rightarrow \infty$, with $f(u) / u$ increasing.

The paper is organized as follows: Section 2 deals with some existence results for the problem with a constant weight in balls and annuli, and some comparison lemmas. Section 3 is devoted to the proof of the key local estimates, and of Theorems 1 and 2 , 


\section{Preliminary Results}

As already stated in the introduction, the key to the local estimates for solutions to (1.1) is a rescaling argument based in solutions in balls and annuli. We collect in this section the results needed. Although we are stating more general results than needed, we are only sketching the proof of existence, which is the principal feature we are using.

We begin by recalling a by now well-known result on problem (1.1) when $a(x)=1$ and the domain $\Omega$ is the unit ball $B$ of $\mathbb{R}^{N}$.

Lemma 3. The problem

$$
\left\{\begin{array}{ccc}
\Delta u=u^{p} & \text { in } & B \\
u=+\infty & \text { on } & \partial B
\end{array}\right.
$$

has a unique positive solution $U \in C^{\infty}(B)$, which is radially symmetric. Moreover,

$$
\lim _{r \rightarrow 1-}(1-r)^{\alpha} U(r)=(\alpha(\alpha+1))^{\frac{1}{p-1}}
$$

where $\alpha=2 /(p-1)$.

Proof. As already claimed, we only show existence. For $n \in \mathbb{N}$, consider the problem

$$
\left\{\begin{array}{ccc}
\Delta u=u^{p} & \text { in } & B, \\
u=n & \text { on } & \partial B .
\end{array}\right.
$$

Since $\underline{u}=0$ is a subsolution while $\bar{u}=n$ is a supersolution, it follows that (2.3) admits a solution $u$. According to regularity theory, $u \in C^{\infty}(\bar{B})$ (cf. [16]). This solution is moreover unique thanks to the maximum principle, since the right-hand side in (2.3) is increasing. It will be denoted by $u_{n}$. Uniqueness also implies that $u_{n}$ is radially symmetric, and increasing in $n$.

We now claim that the solutions are uniformly bounded in compacts of $B$. To show this, it suffices with constructing a suitable supersolution. It is not hard to see that

$$
\bar{u}=C\left(1-|x|^{2}\right)^{-\alpha}
$$

where $C$ is large enough is a convenient supersolution, and a comparison argument gives $u_{n} \leq \bar{u}$ for all $n$. This shows the claim.

Since the solutions are uniformly bounded in compacts of $B$, it is standard to pass to the limit and obtain that $u_{n} \rightarrow U$ in $C^{2}(B)$, where $U$ is a positive solution to (2.1). By regularity theory it follows again that $U \in C^{\infty}(B)$.

We next turn to consider a variant of problem (1.1) with $a(x)=1$, posed in an annulus of $\mathbb{R}^{N}$. For this aim, set $A=\left\{x \in \mathbb{R}^{N}: 1<|x|<3\right\}, \Gamma=\left\{x \in \mathbb{R}^{N}\right.$ : $|x|=1\}, \Gamma^{\prime}=\left\{x \in \mathbb{R}^{N}:|x|=3\right\}$.

Lemma 4. The problem

$$
\left\{\begin{array}{lll}
\Delta v=v^{p} & \text { in } & A, \\
v=+\infty & \text { on } & \Gamma, \\
v=0 & \text { on } & \Gamma^{\prime}
\end{array}\right.
$$

has a unique positive solution $V \in C^{\infty}\left(A \cup \Gamma^{\prime}\right)$, which is radially symmetric. Moreover,

$$
\lim _{r \rightarrow 1+}(r-1)^{\alpha} V(r)=(\alpha(\alpha+1))^{\frac{1}{p-1}}
$$


Proof. To prove existence, consider for a positive integer $n$, the problem

$$
\left\{\begin{array}{ccc}
\Delta v=v^{p} & \text { in } & A, \\
v=n & \text { on } & \Gamma, \\
v=0 & \text { on } & \Gamma^{\prime} .
\end{array}\right.
$$

This problem has a positive solution $v_{n} \in C^{\infty}(\bar{A})$, since $\underline{u}=0$ is a subsolution while $\bar{u}=n$ is a supersolution. The maximum principle implies that $v_{n}$ is unique, and uniqueness implies also that $v_{n}$ is radially symmetric and increasing in $n$.

It is also not hard to check that the function $\bar{v}=C\left(|x|^{2}-1\right)^{-\alpha}$ is a supersolution for large $C$. Thus $v_{n} \leq \bar{v}$, and this implies that $v_{n}$ is locally bounded independently of $n$. Thus $v_{n} \rightarrow V$ in $C_{\mathrm{loc}}^{2}\left(A \cup \Gamma^{\prime}\right)$, where $V$ is a solution to (2.4), which is in addition radially symmetric. Finally, standard elliptic regularity gives $V \in C^{\infty}\left(A \cup \Gamma^{\prime}\right)$, as we wanted to show.

We finally consider for their use in Section 3 two comparison results which are easily obtained by means of a rescaling.

Lemma 5. Let $w \in W^{2, q}(B) \cap L^{\infty}(B)$ verify $\Delta w \geq C w^{p}$ in $B$. Then $w \leq C^{-\frac{1}{p-1}} U$ in $B$, where $U$ is the unique solution to (2.1) given by Lemma 3 .

Proof. It is easily seen that $\underline{u}=C^{\frac{1}{p-1}} w$ is a subsolution to (2.1). Also, since $w \in L^{\infty}(B)$, it follows that $\underline{u} \leq n$ in $B$ for large $n$, and thanks to uniqueness of solutions to (2.3) we have $\underline{u} \leq u_{n}$ for large $n$. The conclusion is clear once we let $n \rightarrow \infty$.

Lemma 6. Let $Q$ be a subset of $A$, and set $\Gamma_{1}=\partial Q \cap(A \cup \Gamma), \Gamma_{2}=\partial Q \cap \Gamma^{\prime}$. Assume $v \in W_{\mathrm{loc}}^{2, q}(Q)$ verifies $\Delta v \leq C v^{p}$ in $Q$, with $v=\infty$ on $\Gamma_{1}$ and $v>0$ on $\Gamma_{2}$. Then $v \geq C^{-\frac{1}{p-1}} V$ in $Q$, where $V$ is the unique solution to (2.4) given by Lemma 4.

Proof. It goes essentially as in Lemma 5. The function $\bar{v}=C^{\frac{1}{p-1}} v$ is a supersolution to $\Delta z=z^{p}$ in $Q$. Since $\bar{v}>v_{n}$ on $\partial Q$, where $v_{n}$ is the unique solution to (2.6), we arrive at $\bar{v} \geq v_{n}$ in $Q$. The lemma is proved by letting $n \rightarrow \infty$.

\section{Proofs}

In this section, we are finally proving Theorems 11 and 2 . As remarked in the introduction, the key to their proofs is the obtention of local estimates for solutions. We begin with the upper estimate, which is an interior one, and does not depend on the regularity of the domain or even on $a(x)$ on $\partial \Omega$ (this estimate is also valid for unbounded weights; see [5]). For $x \in \Omega$, we let $B_{x}=B(x, d(x) / 2)$. Notice that for $x \in \Omega_{\delta / 2}$, we have $B_{x} \subset \subset \Omega_{\delta}$, and hence $\inf _{B_{x}} a>0$. Then:

Lemma 7. Let u be a positive strong solution to (1.1). Then there exists a constant $C>0$ such that for every $x \in \Omega_{\delta / 2}$ we have

$$
u(x) \leq C\left(\inf _{B_{x}} a\right)^{-\frac{1}{p-1}} d(x)^{-\alpha}
$$

where $\alpha=2 /(p-1)$, and $C$ is a constant which depends only on $p$. 


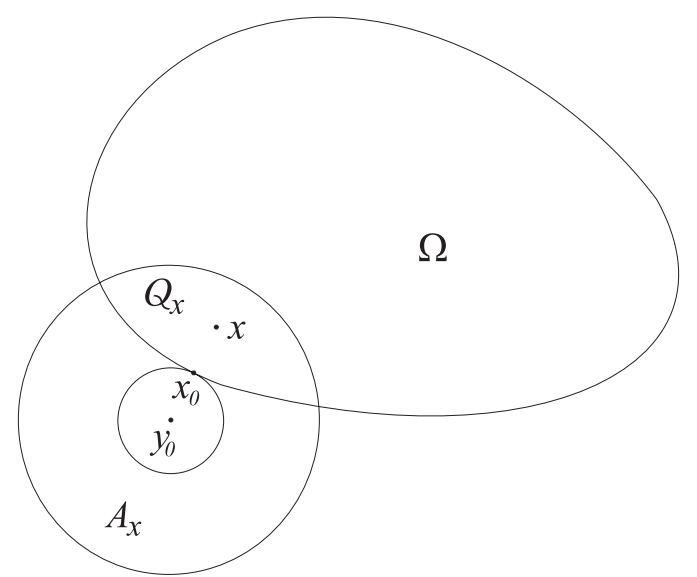

Figure 1. The annulus $A_{x}$ and the subdomain $Q_{x}$ for a point $x \in \Omega$.

Proof. Fix $x \in \Omega_{\delta / 2}$, so that $B_{x} \subset \subset \Omega_{\delta}$, and introduce the function $w(y)=$ $d(x)^{\alpha} u\left(x+\frac{d(x)}{2} y\right)$ for $y \in B$. Then

$$
\Delta w=\frac{1}{4} a\left(x+\frac{d(x)}{2} y\right) w^{p} \geq \frac{1}{4}\left(\inf _{B_{x}} a\right) w^{p}
$$

in $B$. It follows by Lemma 5 that $w(y) \leq 4^{\frac{1}{p-1}}\left(\inf _{B_{x}} a\right)^{-\frac{1}{p-1}} U(y)$ in $B$, where $U$ is the unique positive solution to (2.1). Taking $y=0$, we arrive at $u(x) \leq$ $C\left(\inf _{B_{x}} a\right)^{-\frac{1}{p-1}} d(x)^{-\alpha}$. This completes the proof.

For the lower estimate, we need some regularity of the domain. It turns out that an exterior sphere condition is enough, although we are also assuming - to simplify the presentation - that $\Omega$ is smooth enough to have an outward pointing unit normal field $\nu$. For a point $x \in \Omega_{\delta / 2}$ we denote by $x_{0}$ its projection onto $\partial \Omega$ and set $y_{0}=x_{0}+d(x) \nu\left(x_{0}\right), A_{x}=\left\{y \in \mathbb{R}^{N}: d(x)<\left|y-y_{0}\right|<3 d(x)\right\}$, $Q_{x}=A_{x} \cap \Omega$. Notice that the annulus $A_{x}$ is tangent to $\partial \Omega$ at $x_{0}$, while $x \in A_{x}$ (see Figure 1). Then we have:

Lemma 8. Let $u$ be a positive strong solution to (1.1). There exists a positive constant $C$ such that for every $x \in \Omega_{\delta / 2}$

$$
u(x) \geq C\left(\sup _{Q_{x}} a\right)^{-\frac{1}{p-1}} d(x)^{-\alpha} .
$$

Proof. Set $Q=\left\{y \in A: y_{0}+d(x) y \in Q_{x}\right\}$, and $v(y)=d(x)^{\alpha} u\left(y_{0}+d(x) y\right)$, where $y \in Q$. Then $v$ verifies

$$
\Delta v=a\left(y_{0}+d(x) y\right) v^{p} \leq\left(\sup _{Q_{x}} a\right) v^{p}
$$

in $Q$. Now notice that $v=\infty$ on $\partial Q \cap\left(A \cup \Gamma\right.$ ), while $v>0$ on $\partial Q \cap \Gamma^{\prime}$ (we are using the notation of Section 2). Hence, by Lemma 6 we arrive at $v \geq\left(\sup _{Q_{x}} a\right)^{-\frac{1}{p-1}} V$ in $\tilde{A}_{x}$.

In particular, take $y=-2 \nu\left(x_{0}\right)$, so that $y_{0}+d(x) y=x$. Then we have $u(x) \geq$ $C\left(\sup _{Q_{x}} a\right)^{-\frac{1}{p-1}} d(x)^{-\alpha}$, where $C$ does not depend on $x$. 
Remark 2. We observe for subsequent use that $\frac{1}{2} d(x) \leq d(y) \leq \frac{3}{2} d(x)$ for $y \in \overline{B_{x}}$, while $d(y) \leq 2 d(x)$ for $y \in \overline{Q_{x}}$.

As a byproduct of the estimates contained in Lemmas 7 and 8 , we can now prove the boundary estimates in Theorem 2 .

Proof of Theorem 2. It suffices to prove that for every sequence $\left\{x_{n}\right\} \subset \Omega$ such that $x_{n} \rightarrow \partial \Omega$, we have

$$
C_{1} \leq a\left(x_{n}\right)^{\frac{1}{p-1}} d\left(x_{n}\right)^{\alpha} u\left(x_{n}\right) \leq C_{2} .
$$

Without loss of generality, assume $x_{n} \rightarrow x_{0} \in \partial \Omega$. According to Lemma 7 we have

$$
a\left(x_{n}\right)^{\frac{1}{p-1}} d\left(x_{n}\right)^{\alpha} u\left(x_{n}\right) \leq C\left(\frac{a\left(x_{n}\right)}{\inf _{B_{n}} a}\right)^{\frac{1}{p-1}},
$$

where $B_{n}=B_{x_{n}}$ and $C$ does not depend on $n$. By the continuity of $a$, there exist points $\eta_{n} \in \overline{B_{n}}$ such that $a\left(\eta_{n}\right)=\inf _{B_{n}} a$. Moreover, $\eta_{n} \rightarrow x_{0}$, and $d\left(\eta_{n}\right) \geq \frac{1}{2} d\left(x_{n}\right)$ (Remark 2), so that $d\left(x_{n}\right) / d\left(\eta_{n}\right) \leq 2$. Hence, by Condition (A)

$$
a\left(x_{n}\right)^{\frac{1}{p-1}} d\left(x_{n}\right)^{\alpha} u\left(x_{n}\right) \leq C\left(\frac{a\left(x_{n}\right)}{a\left(\eta_{n}\right)}\right)^{\frac{1}{p-1}} \leq C_{2},
$$

where $C_{2}$ does not depend on $n$. This proves the rightmost inequality in (3.1). The leftmost inequality is proved similarly, but with the use of Lemma 8 instead.

We can finally proceed to the proof of Theorem 1. We first remark that, thanks to the proof of Theorem 2, given any two positive strong solutions $u, v$ to (1.1), it follows that $u / v$ is bounded and bounded away from zero.

Proof of Theorem 1. Existence is a consequence of Theorem 1 in [20], although the solutions are not classical, as stated there, but only strong. Thus we only show uniqueness.

The proof is a refinement of the iterative argument in [18] (see also [5]). Let $u$, $v$ be positive solutions to (1.1). As already pointed out, the function $w=u / v$ is bounded from above. Let $\theta=\sup w$, and assume $\theta>1$.

We claim that for every sufficiently large positive integer $n$, there exists $x_{n}$ such that $x_{n} \in \Omega_{\delta}$ and $u\left(x_{n}\right)>\theta_{n} v\left(x_{n}\right)$, where we are denoting $\theta_{n}=\theta-1 / n$. Indeed, it is not hard to see that $w$ verifies the equation

$$
v \Delta w+2 \nabla v \nabla w+a(x) v^{p} w\left(1-w^{p-1}\right)=0 .
$$

If we denote by $\Omega_{0}=\left\{x \in \Omega: w(x)>\theta_{n}\right\}$ and assume that $\Omega_{0} \subset \subset \Omega$, we obtain by means of the maximum principle that $w \leq \theta_{n}$ in $\Omega_{0}$, which is a contradiction. Thus, $\partial \Omega_{0} \cap \partial \Omega \neq \emptyset$, and the claim follows. Notice that this proof also shows that we can select $x_{n}$ so that $d\left(x_{n}\right) \rightarrow 0$.

With no loss of generality we may assume $x_{n} \rightarrow x_{0} \in \partial \Omega$. Now introduce the set $D=\Omega_{0} \cap B_{n}$, where $B_{n}=B_{x_{n}}$, and denote $r=d\left(x_{n}\right) / 2$ (we are not expressing the dependence of $r$ on $n$, since it is only a temporary variable). In $D$ we have

$$
\Delta\left(u-\theta_{n} v\right)>C \theta_{n} a(x) v^{p},
$$

where $C$ will denote during the rest of the proof a positive constant which is bounded away from zero for large $n$. We now use the estimates provided by Lemma 8 , and 
obtain that

$$
\Delta\left(u-\theta_{n} v\right)>C \theta_{n}\left(\inf _{B_{n}} a\right)\left(\sup _{Q_{n}} a\right)^{-\frac{p}{p-1}} r^{-\alpha p},
$$

in $D$, where $Q_{n}=Q_{x_{n}}$. Let $\phi$ be the unique solution to $-\Delta \phi=1$ in $B_{n}, \phi=0$ on $\partial B_{n}$. It is well known that $\phi$ is radially symmetric with respect to $x_{n}$, and given by $\phi(x)=C\left(r^{2}-\left|x-x_{n}\right|^{2}\right)$, for some determined positive constant $C$. It follows from (3.2) that

$$
\Delta\left(u-\theta_{n} v+C \theta_{n}\left(\inf _{B_{n}} a\right)\left(\sup _{Q_{n}} a\right)^{-\frac{p}{p-1}} r^{-\alpha p} \phi\right)>0
$$

in $D$, and thanks to the maximum principle, there exists $y_{n} \in \partial D$ such that

$$
\begin{aligned}
u\left(x_{n}\right)-\theta_{n} v\left(x_{n}\right) & +C \theta_{n}\left(\inf _{B_{n}} a\right)\left(\sup _{Q_{n}} a\right)^{-\frac{p}{p-1}} r^{-\alpha p} \phi\left(x_{n}\right) \\
& <u\left(y_{n}\right)-\theta_{n} v\left(y_{n}\right)+C \theta_{n}\left(\inf _{B_{n}} a\right)\left(\sup _{Q_{n}} a\right)^{-\frac{p}{p-1}} r^{-\alpha p} \phi\left(y_{n}\right) .
\end{aligned}
$$

Observe that $\partial D=\left(\partial \Omega_{0} \cap \bar{B}_{n}\right) \cup\left(\bar{\Omega}_{0} \cap \partial B_{n}\right)$. If $y_{n} \in \partial \Omega_{0} \cap \bar{B}_{n}$, that is, $u\left(y_{n}\right)=\theta_{n} v\left(y_{n}\right)$, we obtain $\phi\left(x_{n}\right)<\phi\left(y_{n}\right)$, which is impossible, since $\phi$ is radially decreasing. Thus $u\left(y_{n}\right)>\theta_{n} v\left(y_{n}\right)$, and hence $y_{n} \in \partial B_{n}$. In particular, $\phi\left(y_{n}\right)=0$ and

$$
C \theta_{n}\left(\inf _{B_{n}} a\right)\left(\sup _{Q_{n}} a\right)^{-\frac{p}{p-1}} r^{-\alpha p+2}<u\left(y_{n}\right)-\theta_{n} v\left(y_{n}\right) .
$$

Now by Lemma 7 we have that

$$
v\left(y_{n}\right) \leq C\left(\inf _{B_{n}^{\prime}} a\right)^{-\frac{1}{p-1}} r^{-\alpha},
$$

where $B_{n}^{\prime}=B\left(y_{n}, d\left(y_{n}\right) / 2\right)$. Setting $B_{n}^{\prime \prime}=B_{n} \cup B_{n}^{\prime}$, we arrive from (3.3) at

$$
C \theta_{n}\left(\frac{\inf _{B_{n}^{\prime \prime}} a}{\sup _{Q_{n}} a}\right)^{\frac{p}{p-1}} v\left(y_{n}\right)<u\left(y_{n}\right)-\theta_{n} v\left(y_{n}\right) .
$$

Thanks to the continuity of $a$, there exist $\xi_{n} \in \overline{Q_{n}}, \eta_{n} \in \overline{B_{n}^{\prime \prime}}$ such that $a\left(\xi_{n}\right)=$ $\sup _{Q_{n}} a, a\left(\eta_{n}\right)=\inf _{B_{n}^{\prime \prime}} a$, and clearly $a\left(\xi_{n}\right), a\left(\eta_{n}\right)>0$, while $\xi_{n}, \eta_{n} \rightarrow x_{0}$. Moreover, $d\left(\xi_{n}\right) \leq 2 d\left(x_{n}\right), d\left(\eta_{n}\right) \geq \frac{3}{4} d\left(x_{n}\right)$, so that $d\left(\xi_{n}\right) / d\left(\eta_{n}\right) \leq 8 / 3$. Thus by Condition (A) we finally have from (3.4) that $u\left(y_{n}\right)>\theta_{n}(1+C) v\left(y_{n}\right)$, for a constant $C$ which is bounded away from zero for large $n$. Taking into account that $u\left(y_{n}\right) \leq \theta v\left(y_{n}\right)$, we achieve $\theta>\theta_{n}(1+C)$. After letting $n$ go to infinity, we have $\theta \geq \theta(1+C)$, which is a clear contradiction.

This contradiction proves that $\theta \leq 1$, that is, $u \leq v$, and the symmetric argument gives $u=v$. This concludes the proof.

\section{REFERENCES}

[1] C. Bandle, M. Marcus, Sur les solutions maximales de problèmes elliptiques non linéaires: bornes isopérimetriques et comportement asymptotique, C. R. Acad. Sci. Paris Sér. I Math. 311 (1990), 91-93. MR1065436 (91f:35096)

[2] C. Bandle, M. Marcus, 'Large' solutions of semilinear elliptic equations: Existence, uniqueness and asymptotic behaviour, J. Anal. Math. 58 (1992), 9-24. MR.1226934 (94c:35081)

[3] L. Bieberbach, $\Delta u=e^{u}$ und die automorphen Funktionen, Math. Ann. 77 (1916), 173-212. MR1511854

[4] M. Chuaqui, C. Cortázar, M. Elgueta, C. Flores, J. García-Melián, R. Letelier, on an elliptic problem with boundary blow-up and a singular weight: the radial case, Proc. Roy. Soc. Edinburgh 133 (2003), 1283-1297 MR2027646 (2005b:35074) 
[5] M. Chuaqui, C. Cortázar, M. Elgueta, J. García-Melián, Uniqueness and boundary behaviour of large solutions to elliptic problems with singular weights, Comm. Pure Appl. Anal. 3 (2004), 653-662. MR.2106305 (2005h:35115)

[6] F. Cîrstea, Y. Du, General uniqueness results and variation speed for blow-up solutions of elliptic equations, Proc. London Math. Soc. 91 (2005), 459-482. MR2167094(2006e:35123)

[7] F. Cîrstea, V. RĂDulescu, Uniqueness of the blow-up boundary solution of logistic equations with absorbtion, C. R. Acad. Sci. Paris Sér. I Math. 335 (5) (2002), 447-452. MR1937111 (2003i:35102)

[8] F. CîRSteA, V. RĂdulescu, Nonlinear problems with boundary blow up: a Karamata regular variation theory approach, Asymptotic Anal. 46 (2006), 275-298. MR2215886|(2007a:35045)

[9] M. Del Pino, R. Letelier, The influence of domain geometry in boundary blow-up elliptic problems, Nonlinear Anal. 48 (6) (2002), 897-904. MR1879080 (2002m:35075)

[10] G. Díaz, R. Letelier, Explosive solutions of quasilinear elliptic equations: Existence and uniqueness, Nonlinear Anal. 20 (1993), 97-125. MR.1200384 (94a:35017)

[11] Y. Du, Asymptotic behavior and uniqueness results for boundary blow-up solutions, Diff. Int. Eqns. 17 (2004), 819-834. MR2074688 (2005f:35096)

[12] Y. Du, Q. HuAng, Blow-up solutions for a class of semilinear elliptic and parabolic equations, SIAM J. Math. Anal. 31 (1999), 1-18. MR1720128 (2000g:35059)

[13] J. García-Melián, Nondegeneracy and uniqueness for boundary blow-up elliptic problems, J. Diff. Eqns. 223 (2006), 208-227. MR2210144

[14] J. García-Melián, R. Letelier-Albornoz, J. Sabina de Lis, Uniqueness and asymptotic behaviour for solutions of semilinear problems with boundary blow-up, Proc. Amer. Math. Soc. 129 (2001), 3593-3602. MR1860492 (2002j:35117)

[15] J. García-Melián, J. SABina DE Lis, Remarks on large solutions, in "Spectral Theory and Nonlinear Analysis with Applications to Spatial Ecology". S. Cano-Casanova, J. LópezGómez, C. Mora-Corral Eds. World Scientific Publishing, Singapore, 2005.

[16] D. Gilbarg, N.S. Trudinger, Elliptic partial differential equations of second order, Springer-Verlag, 1983. MR0737190 (86c:35035)

[17] J. B. Keller, On solutions of $\Delta u=f(u)$, Comm. Pure Appl. Math. 10 (1957), 503-510. MR0091407 (19:964c)

[18] S. KIM, A note on boundary blow-up problem of $\Delta u=u^{p}$, IMA preprint No. 1872, (2002).

[19] V. A. Kondrat'ev, V. A. Nikishinin, Asymptotics, near the boundary, of a solution of a singular boundary value problem for a semilinear elliptic equation, Differential Equations 26 (1990), 345-348. MR.1053773 (91g:35048)

[20] A. V. LAIR, A. W. Wood, Large solutions of semilinear elliptic problems, Nonlinear Anal. 37 (1999), 805-812. MR1692803 (2000d:35075)

[21] C. Loewner, L. Nirenberg, Partial differential equations invariant under conformal of projective transformations, in "Contributions to Analysis (a collection of papers dedicated to Lipman Bers)", Academic Press, New York, 1974, p. 245-272. MR0358078 (50:10543)

[22] J. López-Gómez, The boundary blow-up rate of large solutions, J. Diff. Eqns. 195 (2003), 25-45. MR 2019241 (2004k:35122)

[23] J. LóPEz-Gómez, Optimal uniqueness theorems and exact blow-up rates of large solutions, J. Diff. Eqns. 224 (2006), 385-439. MR2223723

[24] M. MARCUs, L. VÉRON, Uniqueness and asymptotic behaviour of solutions with boundary blow-up for a class of nonlinear elliptic equations, Ann. Inst. H. Poincaré Anal. Non Linéaire 14 (1997), 237-274. MR1441394 (97m:35068)

[25] L. VÉRon, Semilinear elliptic equations with uniform blowup on the boundary, J. Anal. Math. 59 (1992), 231-250. MR1226963 (94k:35113)

[26] Z. Zhang, A remark on the existence of explosive solutions for a class of semilinear elliptic equations, Nonlinear Anal. 41 (2000), 143-148. MR.1759143(2001b:35099)

Departamento de Análisis Matemático, Universidad de la laguna, C/ Astrofísico Francisco Sánchez s/n, 38271, La Laguna, Spain

E-mail address: jjgarmel@ull.es 